In slight forms of unnatural stools, increase the dilution of the top milk, and reduce the quantity of sugar slightly. If large lumps of fat are in the stools, use milk containing less fat for diluting; this can be obtained by taking more top milk out of the quart bottle. Where lumps of casein are apparent, the diluent must be increased. If increasing the dilution and reducing the fat and sugar does not overcome the trouble, stop the milk and feed dextrinized gruel for a day, gradually adding a little milk, which is increased in amount as fast as the infant can digest it.

There are times when infants can not digest milk in any form, no matter how much it may be diluted, nor what diluent is employed. They may then be given mutton broth, from which the fat has been removed, extracted beef-blood and water, dextrinized wheat or barley gruel, or dextrinized gruel, to which either the white or yolk of an egg has been added.

The following will show the food principles obtained by such egg mixtures:

Dextrinized gruel and white of egg.-Dextrinized wheat-flour gruel, 8 fluidounces, white of one egg-581 grains (large egg).

About 2 per cent. proteids (egg 1.25, gruel .75), 4 per cent. soluble carbohydrates.

Putting two even spoonfuls of granulated sugar into this mixture, 3 per cent. of sugar is added, which, with the 4 per cent. of digested starch, makes $r$ per cent. total soluble carbohydrates.

White of egg contains 85 to 88 per cent. water; .07 per cent. salts; 10 to 13 per cent. proteid bodies, made up of albumin, gobulin and mucoid substance and traces of fats, lecithin and cholesterin.

Dextrinized gruel and yolk of egg.-Dextrinized wheat-flour gruel, 8 fluidounces, yolk of one egg-288 grains (large egg.)

About fat 1.7, proteids 1.\%, (egg 1., gruel .ro) and 4 per cent. soluble carbohydrates.

By putting two even spoonfuls of granulated sugar into this mixture, 3 per cent. of sugar is added, which with the 4 per cent. of digested starch, makes 7 per cent. total soluble carbohydrates.

Yolk of egg contains about 47.19 per cent. water and 52.81 per cent. solids, made up of 15.63 per cent. proteids, .96 per cent. salts, 22.84 per cent. fat, 10.7 per cent. lecithin and 1.75 per cent. cholesterin. Proteids of yolk contain phosphorus and iron, which is of great importance in the formation of blood, and a pseudonuclein which is also found in casein of milk. These are not present in the proteids of the white of egg. Lecithin, which also contains phosphorus, is undoubtedly "a very important material for building up of the complicated phosporized nuclein substance of the cell and cell nucleins." Lecithin is also found in milk, especially woman's milk.

\section{THE SPECIFIC TREATMENT OF CROUPOUS PNEUMONIA.*}

WIIJISON O. BRIDGES, M.D.

OMAHA, NEB.

The mortality of croupous pneumonia is greater than that of any other acute disease; higher than that of diphtheria, and next below that of tuberculosis. The mortality statistics in private and hospital practice show a percentage of from twenty to forty. The census of 1890 indicated that 76,496 persons died of pneumonia 1900 . in the United States during the preceding year, a deathrate of 186.94 per 100,000 of population. The census reports of 1870,1880 and 1890 show that the deaths from this disease have slightly increased. It is the most wide-spread of all acute diseases.

It is nearly twenty years since Fränkel discovered the diplococcus lanceolatus and established its causative relation to croupous pneumonia, since which time renewed efforts have been, and are being, made for the discovery of a specific prophylactic and curative agent. Some years ago the Klemperers demonstrated that the injection of blood-serum, obtained from one who had recently recovered from pneumonia, conferred immunity in some of the lower animals, and it was hoped that an antitoxic serum would be obtained which would be prophylactic and curative in man. While some favorable results from the injection of 20-c.c. doses have bern reported by a number of observers, the treatment has not as yet gained a foothold. Various drugs have been from time to time advocated as specifics; long before the antimicrobic theory of treatment was suggested, calomel in large doses-20 to 40 grains-quinin in amounts of from 30 to 50 grains daily, veratrum viride in doses sufficient to bring and maintain the pulse below sixty, had the endorsement of many competent observers whose reports were certainly commendatory. The same may be said of venesection, which at one time was supposed to be the treatment par excellence. To-day the teaching is that the expectant plan with the meeting of indications by appropriate symptomatic remedies yields as good results as have heretofore been obtained; and there is much less probability of doing harm. Osler states in his 1899 edition that "Pneumonia is a selflimited disease which can neither be aborted nor cut short by any known means at our command. Even under the most unfavorable circumstances it may terminate abruptly and naturally without a dose of medicine having been administered. There is no specific treatment for pneumonia. The young practitioners may bear in mind that patients are more often damaged than helped by the promiscuous drugging which is still only too prevalent." The remedies advocated as specifics before the expectant plan was adopted were selected because of their antiplastic, antiphlogistic and antipyretic properties. Pneumonia was then supposed to be an inflammatory disease and was to be combated like any other inflammation. Calomel acquired its adherents because of its supposedly antiplastic effects, veratrum viride, on account of its antiphlogistic properties, and quinin because of its power over fever.

Flint made a long step in advance, when he announced his conviction that pneumonia was not simply an inflammatory disease of the lungs, but an essential infectious fever with pulmonic inflammatory manifestations. $\mathrm{He}$ it was who advocated large doses of quinin on this hypothesis. He writes, "As long ago as 1861 I was led, by the results of the analysis of a considerable number of cases in which sulphate of quinin was given to the extent of only 15 grains daily,to the conclusion that this remedy exerted a marked causative influence on the disease. I can now (1881) bear testimony to the fact that given in large doses, namely 20 to 30 grains daily, this remedy in a certain proportion of cases renders the disease abortive, and that when this does not follow, the disease is often modified to a greater degree than by smaller doses. Now, whatever efficacy belongs to the remedy proceeds evidently not from any direct effect upon the pul- 
monary affection, but from a controlling influence over the pyrexia, thus sustaining the doctrine that the disease is an essential fever."

'The newer pathology of a pneumococcus infection was but a step in advance of Flint's doctrine, for it determined the actual cause of the infection. It holds that the disease is a general toxemia-resulting from the formation of toxins, produced by the development of the diplococcus in the lung, having a varying effect on the nervous system, the circulation, and the digestive tract, plus an inflammatory condition of the pulmonary vesicular walls and smaller bronchi, the direct irritative effect of the bacterial growth, which results in an exudate filling the air-vesicles and thus interfering with the functional pulmonary circulation and respiration by mechanical pressure. With what we believe now to be the true pathology of croupous pneumonia, may we not hope that a true specific will be found, or are we to calmly sit by the bedside and study the interesting clinical phenomena awaiting the crisis or a fatal termination with only symptomatic treatment? The true specific which shall be applicable to all cases must either inhibit the development of the pneumococcus in the lung-by directly destroying it or by rendering its environment inimical to its growth-or act as an antidote to the toxins produced. It must at the same time be innocuous. With these thoughts in mind I treated a severe case of embolic pneumonia of streptococcus origin nearly two years ago, by means of large doses of guaiacol carbonate, with good results, and was on this account deeply interested in a paper on "The Treatment of Pneumonia as Based Upon Recent Views as to Its Pathology," by Andrew H. Smith, of New York, and published in the Medical News, Dec. 6, 1899, in which he urges very strongly the use of guaiacol carbonate or the salicylates as a specific in this disease. He refers to Robinson and Kerr, who are recent enthusiastic advocates of the use of creosote both by the stomach and by inhalation. The great advantage of this remedy is that it can be taken in large doses without harm and that, being eliminated in part through the lungs, it has a double action. E. Congier in an inaugural address published in 1898 , showed that the carbonate can be taken in very large doses up to a dram or more, without any irritation of the stomach, and that being slowly decomposed in the intestine, it liberates the creosote so that the blood is constantly charged with it even when there are six to twelve hour intervals between the doses. Thirteen cases from the clinic of Dr. Cassonte, of Marseilles, are reported in this paper, three of lobar and ten of catarrhal pneu. monia, in which the carbonate was practically the only remedy employed. In every instance the effect upon the temperature was most marked, defervescence occurring promptly in the cases of lobar pneumonia, while in the catarrhal the severity and the duration of the attack were proportionately lessened. Dr. Wm. H. Thomson, of New York, in the discussion of Dr. Smith's paper before the New York Academy of Medicine, said, "Recently, in cases of double pneumonia, I have had excellent success with 20-grain doses of creosote carbonate administered every two hours. A very palatable emulsion can be made of the drug and patients take it readily. I have had three most unpromising double pneumonias recently recover under this treatment."

Robert Liegel, in 1898, reported seventy-two consecutive cases of recovery from pneumonia under the salicylate treatment. The patients were from 16 to 74 years of age. Eight had emphysema, six cardiac disease and a large proportion were alcoholics. The drug used was the sodium salicylate in large doses, not less than 120 grains daily. No other drug was used excepting an expectorant and small doses of morphin when pain was excessive. Under this treatment not only did recovery ensue in every case, but the duration of the attack was materially lessened and in no case did crisis occur, the temperature declining after the first day, reaching the normal by the fifth, when convalescence was established. The expectoration soon became catarrhal and the physical signs did not fully develop, or where they did rapidly retrogressed. In the first cases treated the remedy was suspended too soon, and a relapse occurred. After this was observed the drug was continued several days after convalescence, when no further tendency to relapse was manifested. Previous to the adoption of this treatment the management of the disease had been unsatisfactory and the mortality high. Liegel believes that the salicylate exerts a specific effect on the mucous membrane, increasing its secretion and throwing off the exudate. Dr. Smith believes that Liegel did not grasp the antibacterial idea of the action of the drug. He states that his own experience with this line of treatment has been encouraging, though not large. $\mathrm{He}$ has used all of the drugs which have appealed to him as specifics, with the exception of quinin, which has seemed too slow in action and too distressing to the stomach. He prefers the creosote carbonate to the salicylates, because of the gastric irritation often produced by the latter and its depressing effect upon the heart in the later stage of the disease. So enthusiastic is Dr. Smith in his belief of the efficacy of this plan of treating pneumonia that he urges the family physician "to provide his families with ereosotal or one of the salicylates and to instruct them that with the slightest chilly sensation or the slightest stabbing pain in the lung, a dose is to be given at once, and that this dose is to be repeated at stated intervals until the physician arrives. This might prevent the development of pneumonia in some cases and shorten the attack in others."

Have we a specific for pneumonia in these two drugs, and by this I mean a* remedy which will apply to all cases on antibacterial grounds, which will do no harm and which will not in any way interfere with the treatment of symptoms? This question I have attempted to solve to my own satisfaction during the past winter when I have had an opportunity to put it in practice, and $I$ append a few illustrative cases, some of which were exceedingly severe.

CASE 1.-Mr. H., aged 35, single, accountant, had pneumonia several years before. When in apparent good health, he was seized with a shaking chill lasting one-half hour attended by severe pain under right nipple and a slight cough. Recalling his former experience he at once went to bed and I was summoned. On reaching him two hours later I found him with acute pain in the right side, temperature 101, pulse 96 , respiration 24. During my visit he coughed and expectorated the typical rusty sputum. On physical examination there was diminished expansion of the right side, lessened pulmonary resonance in the lower right mammary region, and characteristic crepitant râles over a part of this area. He was given a prolonged hot bath, 5 grains of calomel, followed by a saline; a mustard and flax-seed poultice was applied to the chest and 15-gr. doses of salicylate of sodium were administered every two hours; and I enjoined absolute rest in bed after the bath, and liquid diet. In eighteen hours the pain was much less, he sweated freely, his temperature was 99.5, pulse 90, respiration 24, no further physical signs. Several 
times he had raised bloody sputum. Salicylate intervals were increased to three hours. In twenty-four hours later the temperature was normal, pulse 80 , respiration 20 , sputum only slightly tinged. From this time he went on to complete convalescence, the salicylate being kept up for five days more at longer intervals.

CASE 2.-Miss E., aged 30, single, teacher, three days before my first visit had a severe chill while at work, followed by lancinating pain in the right side, and dry hacking cough with fever, severe nausea and vomiting, the latter occurring several times. On first examination her countenance was flushed and expression anxious; there was severe pain in the right nipple line through the shoulder; her tongue was much furred, and she was exceedingly nervous and sleepless; her temperature was $104 \mathrm{~F}$., pulse 100 , respiration 40 ; the cough was dry and painful, and no sputum had been raised. Physical examination showed much-diminished expansion on the right side, increased vocal fremitus, complete dulness and bronchial respiration with bronchophony over right infrascapular and axillary regions. The treatment consisted in liquid diet, absolute recumbency, calomel 8 grs., followed by a saline; guaiacol carbonate 8 grs., strychnia $1 / 40$ gr. every two hours; codein $1 / 2$ gr. and phenacetin $4 \mathrm{grs}$. for the pain; mustard and flax-seed poultice covered by oiled muslin.

Fourth day.-The patient's temperature was 104, pulse 108 , respiration 52; she was delirious most of the time, and her sputum was rusty. Consolidation of the right lung extended to the mammary region, and there were signs of consolidation in left infrascapular region; the treatment was continued.

Fifth day.-The temperature was 104.2 , pulse 108 , respiration 50; there were delirium, labored breathing and marked cyanosis; oxygen gas was administered for twenty-minute periods every alternate hour.

Sixth Day.-The temperature was 101.4, pulse 106, and respiration 38 .

Seventh day.-The temperature fell from 101.4 to 99.6, the pulse from 106 to 92 , the respiration was 40 .

The termination was by lysis, and convalescence was uninterrupted. The total amount of guaiacnl carbonate given in four days was 320 grains.

CASE 3.-Mr. N., single, aged 28, chemist, previously well, was seized with severe chill, followed by fever, and pain in the right mammary region. I saw him first on the third day, when his temperature was 104, pulse 120, and respiration 40. He had a cough and expectorated the typical rusty sputum; there was slight delirium and his tongue was thickly furred. The physical signs gave evidence of complete consolidation of the upper lobe of the right lung The treatment was: calomel $8 \mathrm{grs}$. followed by a saline; guaiacol carbonate $8 \mathrm{grs}$, and strychnia $1 / 30$ gr. every two hours; absolute recumbency; liquid diet.

Fourth day.-The temperature was 104.5, pulse 120, respiration 50 ; the delirium was most pronounced, and there was much restlessness; the tongue was brown and dry, and the consolidation extended to the lower lobe, also to the lower lobe of the left lung. The guaiacol was increased to $12 \mathrm{grs}$.

Fifth day.-The temperature was 105 , pulse 140 , respiration 60 ; there was muttering delirium, sordes tended to collect on the teeth, and cyanosis was commencing. The right lung was completely solidified, the left lobe showed an increased amount of solidification, the heart sounds were feeble but regular. Oxygen gas treatment was commenced, twenty minutes every alternate hour; strychnia was increased to $1 / 20$ gr. hypodermically every two hours; 8 grs. calomel were followed by a saline.

Sixth day.-The temperature was. 103, the pulse 103, and the respiration 50 ; the delirium was less marked; there was restlessness, but cyanosis was not continuous, the tongue was moist, and there was commencing resolution in the upper right lung. Oxygen gas gave great relief, but the patient wanted it no longer than ten minutes; the treatment was continued.

Seventh day.-The temperature was 101 , the pulse 108 , and the respiration 50; there was no delirium, and the physical signs of resolution in right lung were extended and were com. mencing in left.
Eighth day.-The temperature was 99 , the pulse 100, and the respiration 40 . From now on convalescence was uninterrupted and resolution became complete in three or four days. Guaiacol was continued for one week in lessened doses, as was also strychnia.

CASE 4.-Mrs. M., aged 32, for several years in delicate health, had a chill with fever and severe pain in right axillary region, and a cough with mucopurulent expectoration. Physical signs of consolidation were not manifest until the sixth day, when they were found in the lower lobe of the right lung; her temperature was 103.4, and her pulse 90. Guaiacol carbonate 12 grs., and strychnia $1 / 30$ gr., were administered every two hours. From this time on for six days the temperature ranged from 101.9 to 104.4 , the highest pulse was 120 , the highest respiration 60 ; delirium was marked at times. Resolution took place by lysis, but the temperature did not reach the normal. After one week of improvement the temperature and pulse increased, when physical signs of a pleural effusion were found. An exploratory paracentesis thoracis revealed pus in the infrascapular region after two failure punctures in the mammary and axillary regions. A pleurotomy with rib resection was performed by Dr. Jonas, which was followed by a tardy though continued convalescence, with complete expansion of the lung.

CASE 5.-Mr. H., aged 45, married, clerk, very robust, had a cold and cough for several days while traveling. A chill followed by fever and pain in right side occurred before reach. ing home, and two days before my first visit. I found the temperature 103 , the pulse 110 , and the respiration 36 ; the cough was accompanied by an expectoration, prune-juice in character. There were physical signs of general bronchitis, and consolidation in the right infrascapular region. Guaiacol carbonate 10 grs. and strychnia $I_{/} 20$ gr. were commenced at onee, and 6 grs. calomel were given, followed by a saline. Under this treatment and a mild expectorant, resolution took place by lysis so that by the sixth day the temperature was normal, and the bronchitis slowly disappeared.

CASE 6.-Mr. C., aged 50, married, accountant, was seen in consultation on the fifth day after a severe chill, followed by high fever and some pain in the side. His temperature had ranged to upward of 104 and pulse to 115. At my first visit he was partially comatose, the tongue was brown and dry, and there were sordes on the teeth; there was enormous digtension of the abdomen from gas, and complete consolidation of the right lung. I prescribed calomel 6 grs., to be followed by a saline; sodium salicylate 15 grs. every two hours for four doses, then at longer intervals, and strychnia 1/20 gr. The following morning the temperature had dropped to about 102 , and the pulse. to 112 ; the abdominal distension was relieved, the tongue was moist, but the stupor had increased and cyanosis was manifest. Oxygen gas was commenced in the afternoon. The same evening the pulse was 124 , and the temperature 103; there was incontinence of feces, the coma had increased, but commencing resolution was manifest in the consolidated lung. In spite of large doses of strychnia and digitalis, hypodermically, the patient died comatose the same night.

CASE 7.-Mrs. S., aged 38, housewife, of rather delicate constitution, had been curetted by me on the eighth day after labor, for a sapremia of mild type which had not yielded to uterine irrigation. Her temperature was practically normal and her condition very favorable until the fifth day following, when she had a chill, with headache and general malaise, followed by a temperature of 103.5. The next day severe pain developed in the left inframammary region, which required morphin hypodermically for its relief. A careful physical examination made after the pain was controlled, when the respiratory movements were free, was negative. Fearing the development of pneumonia, at my next visit I took with me 5 -gr. capsules of guaiacol carbonate.

Third day.-The temperature was 103 , pulse 128, respiration 49 : the pain returned and was also present in the supraclavicular region. There was no cough. Physical examination showed consolidation in the left infrascapular region, crepitant râles distinct in inframammary region of the same 
side. I ordered guaiacol carbonate 10 grs., strychnia 1/20 gr. every two hours, and codein as required for the pain.

Fourth day.-The temperature was 103.2 , the pulse 140 and irregular, the respiration 38; perspiration was free and tympanitis marked. Vaginal examination, abdominal examination negative and physical signs the same, but crepitant râles in addition were heard in the supraclavicular left and inframanmary right, but there was no cough.

Fifth day.-Morning temperature was 102 , the pulse 140 , the respiration 44 ; at $5: 30$ p.m. she had a chilly sensation for a half hour with a temperature of 106 following, the pulse was not countable, the respiration 40 ; at 10 p.m. the temperature was 104, pulse 148 , respiration 36 .

Sixth day.-The temperature was 101.4, the pulse 136, and the respiration 24. Signs of resolution were marked over the consolidated area, and no extension where crepitant rales were heard elsewhere. For the first time she expectorated a bloody sputum which on bacteriologic examination by $\mathrm{Dr}$. Lavender was found to contain staphyloccoci, pneumococei and a very few streptococci. During the next few days all evidence of pulmonary trouble disappeared and the temperature, pulse and respiration became normal. The guaiacol was continued in the same dose at longer intervals.

CASE 8.-This was a case of embolic pneumonia, the result of a septicemia from a self-induced abortion at the second month, terminating in abscess and the expectoration of large quantities of pus containing streptococci and staphylococci. The pulmonary infection oceurred in the left mammary region and gave the typical signs of consolidation, with temperature ranging from 102 to 104 and pulse 130 . Large doses of guaiacol carbonate with strychnia was the only medicinal treatment, the patient convalescing in ten days with good results.

This case can not be classified with the ordinary pneumonia of pneumococcus origin, and is recalled because of the apparent influence of the antimicrobic treatment over a septic embolic pneumonia.

Individual statistics bearing upon the treatment of disease carry weight only with him who has been through the personal experience of the foundation for the statistics. It requires a collective investigation to determine whether in reality any progress has been made, and more particularly is this true of pneumonia, a disease which is attended by so many surprises, and the results of which are so uncertain. My own experience with the remedies which are so earnestly advocated by Dr. Smith is so limited that I hesitated to bring the subject before you at this time; yet I was so impressed with the results in some of them, which represent the severe types of double pneumonia that I desired to suggest a trial along the same line, in order that we might the sooner ascertain whether we yet had a specific treatment for this disease. We must go on hoping that we shall accomplish as much in this direction as we have in the treatment of malaria, of syphilis and of diphtheria. It is all well enough to write and talk of treating the individual instead of the disease but when we can do both with the same degree of intelligence, we shall lower the mortality of pneumonia to figures which, to say the least, will not be alarming. So far as we know I would rely upon the guaiacol or salicylates as specifics in pneumonia, giving preference to the former in cases past the stage of congestion, in the enfeebled, or where there were heart lesions. The addition of strychnia to the salicylate treatment obviates in part the depressant effect upon the heart, and so does alcohol. I combine strychnia with the guaiacol also for its influence upon the nervous system, and to prepare the patient for very large doses, if they become necessary. I shall not take up your time with the details of the individual or symptomatic treat- ment, except to say that I have found the hot poultices more agreeable than cold ; that $I$ would resort to venesection when there was an overloaded right heart with threatening symptoms; that digitalis is reserved for an irregular and flagging heart; codein in small doses for the relief of pain and delirium; strychnia in increasing doses and alcohol for enfeebled heart action; calomel and saline for constipation or sluggish portal circulation; that oxygen gas is commenced at the first sign of cyanosis and in quantity sufficient to relieve; and last, but by all means first, the absolute recumbent posture until resolution is established.

\section{APPENDICULAR FISTULA**} JOHN B. DEAVER, M.D.

PHYSICIAN-IN-CHIEF TO THE GERMAN HOSPITAL. PHILADELPiIIA.

The subject of my remarks to-day, appendicular fistula, is such a common sequel to operations for acute appendicitis and is of such great importance to the future welfare of the patient, that I can not too strongly invite your attention to this subject. It is one of the most important sequels to appendicitis operations, for several reasons: on account of its effect on the constitution, the organs it may involve, and the danger in which it may place the patient in regard to future health. That many fistulæ may be avoided, if earlier operative measures were instituted, can not be questioned. This apparently is a very strong presentation to make, but I shall endeavor to show later in the discussion my reasons for making such a statement. I shall discuss this subject by taking up the varieties, the causes and dangris, the means of prevention, and the treatment.

There are two types of fistulæ of appendicular origin - the external and the internal. The external is subdivided into two, the simple and the fecal. The simple fistula is also of two kinds; the first is where the external channel leads from an unhealed abscess cavity and corresponds to the site of the drainage tract. This variety is simply a suppurating sinus, and tends to heal spontaneously. The delay in healing may be due to the presence of an infected ligature or a piece of gauze used for drainage purposes, and which has dropped into the wound and been lost. On the escape of the ligature or the removal of the ganze, closure of the fistula takes place. In the second variety of simple fistula, the lumen of the appendix is in direct communication with, and discharges its contents into, the fistula. The material which is discharged from the fistula is clear mucus. This variety is found when the lumen of the distal portion of the appendix is in direct communication with the fistula, the lumen of the appendix has however become obliterated at some point, or the organ has, from the inflammatory process, become separated from the cecum or in its continuity, thus allowing the secretions of the organ to escape through the fistula.

There are two varieties of the fecal fistula consequent on appendicitis: 1 . Those in which the lumen of the approximal portion of the appendix is in direct communication with the fistula, and from which there is a fecal discharge; this discharge later becomes entirely mucus or but slightly stained with fecal matter. 2 . Those in which the cecum, the ascending colon, the small intestines, or both small and large intestines, are in communication with the fistula; the opening into the

* Presented to the Section on Surgery and Anatomy, at the Fifty-first Annual Meeting of the American Medical Association, held at Atlantic City, N. J., June 5-8, 1900. 